\section{(6) OPEN ACCESS}

PAPER

\title{
Must research benefit human subjects if it is to be permissible?
}

\author{
Daniel Wikler
}

Correspondence to

Dr Daniel Wikler, Mary

B. Saltonstall Professor of

Ethics and Population Health,

Department of Global Health \&

Population 643C FXB, 651

Huntington Avenue, Boston,

MA 02115, USA;

wikler@hsph.harvard.edu

Received 16 March 2016 Revised 27 July 2016 Accepted 7 August 2016 Published Online First 29 August 2016

\begin{abstract}
Must medical experiments with human subjects offer them a 'favourable risk-benefit ratio', that is, more expectation of benefit than harm or burden, if they are to be judged as ethically justified? Ethical justification is easier for experiments that do offer net benefit to subjects, but ethical justification is possible also for some experiments that do not. Basic science experiments with healthy volunteers and 'Phase I' drug trials that seek to determine tolerable dosage levels are routinely approved by ethical review committees; moreover, guidance they receive from government funding agencies specifically asks them to weigh risks to subjects against benefits to subjects and also benefits to those who may benefit from the knowledge gained in the experiment. If a puzzle remains, it is why there remains any assumption that research ethics requires a 'favourable risk-benefit ratio' for the individual research subject.
\end{abstract}

\section{INTRODUCTION}

The ethics of experimentation involving patients is least troubling when participation in the research offers the best prospects for the subjects' future well-being of all available options. This 'favourable risk-benefit ratio' does not obviate the need for informed consent, but this is ordinarily forthcoming once the prospective subject understands what is on offer.

But must an experiment offer patients their best prospect for healthy recovery, in order to be ethically justified? Indeed, might some studies offer greater prospect of harm than benefit, and still be judged ethically sound?

And if an experiment is to be performed not on patients but on healthy volunteers, must any risks be more than offset by prospective benefits to these subjects, in order to be ethically permissible?

The answers to these questions are almost certainly and unequivocally 'No'. Experiments on patients and on healthy volunteers alike may be ethically justified even when the subjects would be likely to fare better by declining to participate, either to choose other options (eg, treatment) or simply to do nothing. Nor is this in any way a secret in the research ethics literature or in government or professional regulations and guidelines.

If this conclusion is both well established and widely and officially recognised, why is it worth devoting an essay to its defence?

\section{DISCUSSION}

The answer is that the contrary thesis-that only research that offers better chances for gain than harm can be judged ethically sound-seems to be widely believed. Several provisions of the World Medical Association's (WMA) 'Declaration of Helsinki', the most influential international research ethics guidelines, seems to support this view. ${ }^{1}$ Principle 3 states that "The Declaration of Geneva of the WMA binds the physician with the words," "The health of my patient will be my first consideration," and the International Code of Medical Ethics declares that, "A physician shall act in the patient's best interest when providing medical care". Principle 8 holds that "While the primary purpose of medical research is to generate new knowledge, this goal can never take precedence over the rights and interests of individual research subjects". Although other provisions seem clearly to envision the possibility that subjects in some experiments might stand to lose more than they might hope to gain, principles 3 and 8 are stated unequivocally.

It is no coincidence that ethical review committees (IRBs, in the USA) are often called 'Committee for the Protection of Human Subjects', even though for research that offers, in prospect, more likelihood of burden than benefit, the way to protect prospective human subjects would be to convince them not to enrol.

A casual acquaintance with the research ethics literature might reinforce this impression. Much of the discussion about risks and benefits is concerned with elaborating our duties to human subjects and with minimising the risks. The possibility of ethically sound research that offers greater prospect of harm than benefit may be acknowledged, but is rarely emphasised or is even buried.

Nevertheless, ethical review committees (some, if not most) do permit investigators to recruit patients and healthy volunteers for experiments that do not offer a 'favourable risk-benefit ratio' to that individual. Moreover, this action is fully compliant with the research ethics guidelines, which most committees cite as sources of guidance. In this brief paper, I cannot attempt an ethical justification of these judgements (though I do endorse them). My aim is to show that they are not heretical.

\section{'Favourable risk-benefit ratio'}

This phrase, which I have placed between quotation marks in the preceding, is reasonably clear, but some elaboration will be useful if we wish to focus on possible differences in ethical principle rather than semantics.

For example, the phrase 'favourable risk-benefit ratio' is ambiguous between two distinct ratios. It is often used in reference to the prospects of gain and 
loss for the individual participant. In other contexts, however, the ratio in question may be that between the prospective burdens for the individual subject and the sum of two sources of benefit: those accruing to the individual subject and those enjoyed by individuals in the future who may benefit from the application of any knowledge gained as a result of the experiment.

Ambiguity presents little problem so long as their meaning is made clear by the context of use, but in this case it may be a source of confusion. Consider two opposing points of view, one insisting that experiments can never be justified unless human subjects stand to gain at least as much as they stand to lose, the other open to the possibility of endorsing an experiment whose subjects stand to lose more than they stand to gain, so long as the experiment has a reasonable chance of offering significant benefits to others by advancing knowledge that might be used to relieve suffering. Both sides can (and do) say that experiments with human subjects are justified only if they offer a 'favourable risk-benefit ratio'. Nevertheless, the appearance of agreement on this point is purely verbal. The risk-benefit ratio to which the italicised phrase refers when endorsed by the first of these opposing viewpoints includes benefits to the human subjects only, and excludes any benefits that might accrue to patients and others in the future as a result of applications of the knowledge gained in the experiment. The ratio referred to by adherents of the second viewpoint is a different ratio, because it includes those potential benefits (if any) to people in the future, resulting from the application of knowledge gained in the experiment. Thus, it is possible that an adherent of the first of these viewpoints would assume that a guideline or rule that includes the italicised phrase would endorse their viewpoint; but this would be completely erroneous if the guideline or rule was referring to the second, wider ratio.

The terms 'risk' and 'benefit' are less clear than they might be. If understood literally, they are odd choices for calculating an ethically relevant ratio, since the former involves probability as well as magnitude (of potential burden), while word 'benefit' does not. In practice, we no doubt understand that this is a façon de parler and that what is literally meant is ex ante net benefit or ex ante net harm, that is, something like the sum of the products of each possible burden (worse health; injury, duress, indignity; anxiety; investment of time, etc, each measured by some common denominator of burden or loss) multiplied by the probability of their occurrence, compared with a similar sum for prospective benefits.

We focus on ex ante burden and benefit, of course, because ethical approval of the research begins before the experiment is initiated. Ethical review committees may insist on a ceiling or threshold limiting prospective harm in some cases (eg, where the likelihood of moderate benefit is high, so that the ratio is positive, but where the human subject might suffer catastrophic harm or loss of life if things go badly).

Those 'benefits' that do not accrue to the individual subjects are often referred to as 'value to society', or 'gain in scientific knowledge'. The use of these terms, when used in efforts to judge whether the burden (if any) on human subjects is justified in view of the likely outcomes, has two drawbacks. First, each is so broad that it is difficult even to imagine how to estimate their magnitude for an experiment under ethical review. Second, they include (if understood literally) outcomes that would not ordinarily be considered relevant in judging the ethics of an experiment with human subjects. A given experiment might, for example, have 'value to society' if they provided jobs, or if the profits that would accrue to a sponsoring pharmaceutical firm proved to be an economic stimulant, but these kinds of gains are rarely if ever cited as justifying the exposure of human subjects to net ex ante harm or burden (ie, the prospect of harm or burden that would exceed any anticipated gain to the individual subject). Similarly, it is difficult to imagine an ethical review committee approving such an experiment if the 'gain in scientific knowledge' were, say, a significant step toward constructing a proof of a theorem in pure mathematics that would have no useful application or other benefits to society, so far as this can be estimated.

For these reasons, our discussion of whether 'value to society' and/or 'gain in scientific knowledge' can justify experiments that fail to offer a 'favourable risk-benefit ratio' to individual human subjects may be more tractable, if we focus our attention on that which is of value to society that may result from the possible gains in scientific knowledge if the study is conducted. Although there are some possible exceptions, the outcome in question is the potential protection from suffering (or the enhancement of well-being) that might be possible for individuals in the future as a result of the application of the scientific knowledge gained by doing the experiment, if there is any. This specification of terms permits an 'apples versus apples' ethical judgement (wellbeing of research subjects vs well-being of future beneficiaries of research), which the frequently used terms 'value to society' and 'gains to scientific knowledge' do not. This refinement of terms, of course, does not in itself tell us what net risk to subjects might be justified by a specified amount of benefit to others in the future. That is an ethical judgement that the members of the ethical review committee are asked to undertake.

I have used the term 'burden' as the catch-all category for adverse outcomes of participation in experiments. As noted, this permits us to compare like with like when it is used in place of 'risk'. It also enables us to take into account a broader range of adverse outcomes, such as reduced privacy or security, than does the more common term 'harm', which is one kind of burden but is not the principal concern in many experiments.

\section{Examples}

Here are examples of experiments with human subjects that do not offer an ex ante net benefit and which are routinely (and appropriately) approved by ethical review committees at institutes and schools of medicine and public health:

- Basic research that involves risks of burden but no anticipated benefit to subjects. ${ }^{2}$ For example, a study of the mechanisms of perception of pain in the hand required subjects to submit to a series of painful stimuli, including moderate electric shocks and placement of hands in very cold water, while wearing an uncomfortable monitoring device. Care was taken to avoid enrolling subjects who may perceive, rightly or wrongly, that their supervisors would be displeased unless they participated.

- 'Phase I' drug testing, in which promising compounds are injected into human volunteers to determine their safety and tolerability. Though the chance of dramatic improvement for participants in these trials cannot be ruled out, these are so unlikely that standard practice in many institutions is to state flatly that participation in these trials will offer no benefit to prospective subjects.

- So-called 'challenge trials' in infectious-diseases research, in which healthy volunteers contract infectious diseases so that scientists can investigate the mechanisms of infection and the early stages of the diseases. For example, volunteers have been asked to place their arms in a chamber filled with mosquitoes that transmit malaria. In most cases, infected 
volunteers are treated for the disease before it becomes intolerable or inflicts lasting damage, but subjects in many of these trials are cautioned to expect substantial discomfort and pain. ${ }^{3}$

- Placebo-controlled trials of surgical techniques that require 'sham surgery', in which invasive procedures that have no anticipated benefit are conducted on controls so that none of the subjects knows whether the procedure under study was performed on them. In some instances, patients who might be candidates for the experimental procedure are stable, and thus have the option of waiting until the trial is performed on others before deciding whether to agree to have the procedure performed on them (as a clinical procedure rather than within a randomised study); and in some of these cases, patients also have the option of engaging surgeons who will perform the procedure immediately (again, as a clinical procedure rather than within a randomised study). In such cases, there is little or no personal gain for those who volunteer to accept the $50 \%$ chance of having sham surgery practiced on them. They might gain from the knowledge produced by the experiment (eg, that the procedure in question is efficacious, or is not), but this knowledge would likewise be available to them if they stood aside, while others stepped forward as volunteers. $^{45}$

Pointing out that we routinely permit studies like these to proceed does not suffice to show that they are ethically justified, and some notable contributors to the literature on research ethics, most prominently Hans Jonas, ${ }^{3} 6$ have offered powerful arguments for restrictions on such experiments that might preclude some or all of these examples. But these analyses, however eloquently argued, have not prevailed. The most influential ${ }^{i}$ document in research ethics today may be the so-called 'Common Rule' (45CFR46), a regulation applying to all agencies of the US government and their grantees. $\$ 46.111$, 'Criteria for IRB approval of research', 7 requires that

1. Risks to subjects are minimised:

- by using procedures which are consistent with sound research design and which do not unnecessarily expose subjects to risk, and

- whenever appropriate, by using procedures already being performed on the subjects for diagnostic or treatment purposes.

2. Risks to subjects are reasonable in relation to anticipated benefits, if any, to subjects, and the importance of the knowledge that may reasonably be expected to result.

- In evaluating risks and benefits, the IRB should consider only those risks and benefits that may result from the research (as distinguished from risks and benefits of therapies subjects would receive even if not participating in the research).

- The IRB should not consider possible long-range effects of applying knowledge gained in the research (eg, the possible effects of the research on public policy) as among those research risks that fall within the purview of its responsibility.

The key word here is the 'and' that occurs in clause (2): benefits to the individual subject are only part of what should be taken into account in ethical review of proposed research. It is noteworthy that this key regulation permits prospective benefits

${ }^{\mathrm{i}}$ Its influence may be due to the volume of research funds it governs, in addition to any ethical or intellectual merit it may possess. to future individuals to be placed on the ethical balance scale; this is encouraged or even required.

The apparently widespread misconception that the regulation requires a 'favourable risk-benefit ratio' for the individual participant is thus a bit of a puzzle. ${ }^{\text {ii }}$

\section{The 'risk-benefit ratio' in research on HIV AIDS cure and long-term remission}

The preceding discussion has been framed in terms of the expectations by the investigators of the prospects for benefit and for harm and other burdens for research subjects. But prospective subjects may see things differently. This may be the case with research seeking a cure or long-term antiretroviral-free remission for HIV/AIDS. Treatment for this condition has advanced so far that HIV-infected individuals can anticipate a normal lifespan, and the side effects of treatment, while not negligible, are judged by most patients to be tolerable. Participation in certain 'AIDS Cure' experiments might expose the subjects to a nontrivial risk of death in exchange for an uncertain chance of becoming permanently HIV negative.

Now that the latter offers little improved chance of survival, it is remarkable that eager volunteers are plentiful. Perhaps they perceive a value in being HIV-free-something apart from its survival value-that the investigators should count as a benefit that belongs on the risk-benefit ethical balancing scale. Perhaps they are motivated by altruism. Or perhaps they are simply making unsound judgements about what would be in their interests.

Would the ethics of an HIV/AIDS cure experiment depend how we explain the apparent enthusiasm of some research subjects for an AIDS cure experiment that the researchers view as offering greater net harm (including a chance of premature death) than net benefit? It might. Scientists and ethical review committees might be unfriendly to a proposed experiment if the most likely explanation for the willingness of some patients to volunteer was that their judgement was unsound. This might be the result, for example, if-despite elaborate efforts to educate potential subjects, not limited to the consent form-patient volunteers continued to harbour false or magical beliefs about the potential benefits to them of the experimental procedure or drug. The same might be true if the potential recruits who were willing to volunteer seemed desperate or suffering from great shame.

These cases would present an unenviable dilemma to scientists and to ethical review committees, if the experiments might yield valuable evidence that would point the way towards a cure for HIV/AIDS, but where these potential subjects were willing and eager to participate, and met the usual criteria for mental competence. The scientists and ethical review committees might correctly believe that if they approve the studies, critics would be unable to point to any clear violation of ethical rules. Yet they and their critics might be concerned that they were able to

${ }^{i i}$ The US Government refers the readers of its advisory on 45CFR46 to The Belmont Report, an essay on ethical principles involved in research with human subjects that was written at the government's request by a group of ethicists. The Report identifies a small number of basic principles upon which ethical guidelines for research might be based, one of which is called 'beneficence'. If one assumes that this principle requires the scientist to be concerned about the well-being primarily (or perhaps solely) of the research participant (as opposed to beneficent concerns for those who suffer from the condition that might be relieved as a result of the experiment), this might explain the misunderstanding in question. The Belmont Report itself, however, does not support this interpretation. 
proceed with the experiments only by exploiting these patients' desperation or shame.

This ethical issue in HIV/AIDS cure research may be acute and troubling, but it is not unique. Those who volunteer for Phase I research, for infectious-diseases challenge trials, and for placebo-controlled surgical trials involving sham surgery always have their own reasons. These may be difficult to understand, particularly for those who believe that science has proved that we always act in our perceived self-interest. Nevertheless, we permit these trials to proceed. In my own view, it is perfectly appropriate to enrol subjects in many of these trials who are motivated by altruism, fairness or reciprocity, but this paper is not the occasion for arguing this point. This paper is addressed to those who may object to HIV/AIDS cure research on the grounds that these trials may not offer a 'favourable risk-benefit ratio' to individual subjects. That objection may be based on an erroneous assumption about research ethics, one that I have sought to identify and refute.

\section{CONCLUSION}

It is entirely understandable and commendable that investigators pause for a long and careful re-thinking of the ethics of AIDS Cure research before taking the expedient option of accepting the prospective subjects' informed consent, that is, their agreement, following extensive education on risks and benefits, to become subjects in the experiments. The physician-scientists do not want to take advantage of a person's ill-considered enthusiasm for a course of action that may not, all things considered, be prudent-especially (but not exclusively) if the most plausible account of the patient's choice is that the patient mistakenly does believe that participation would be prudent.

Whether to accept a given individual's consent and to enrol that individual in the experiment in such cases is an ethically difficult decision that this paper does not attempt to resolve. But it would be useful if those trying to reason their way through this ethical dilemma do not labour under the misunderstanding that approved research practices (in the USA) require that the 'risk- benefit' ratio for research subjects be 'favourable' based on risks and benefits to the participant alone. They need not be. Potential benefits for others belong to the balance scale too.

In summary, those engaged in an ethical evaluation of a proposed trial of a possible cure for HIV/AIDS must carefully estimate a participant's prospective benefits and also the participant's prospects for harm or burden. If the subject stands to gain more than lose, the trial will be easier to justify. But a 'favourable-risk-benefit ratio' for the individual research subject, however desirable, is not an absolute ethical requirement.

Funding National Institute of Allergy and Infectious Diseases (1 R01 Al114617-01A1, 1 R56 Al114617-01).

Competing interests None declared.

Provenance and peer review Commissioned; externally peer reviewed.

Open Access This is an Open Access article distributed in accordance with the Creative Commons Attribution Non Commercial (CC BY-NC 4.0) license, which permits others to distribute, remix, adapt, build upon this work non-commercially, and license their derivative works on different terms, provided the original work is properly cited and the use is non-commercial. See: http://creativecommons.org/ licenses/by-nc/4.0/

\section{REFERENCES}

1 WMA Declaration of Helsinki-Ethical Principles for Medical Research Involving Human Subjects. http://www.wma.net/en/30publications/10policies/b3/index.html (accessed 7 Mar 2016).

2 Miller FG. Ethical issues in research with healthy volunteers: risk-benefit assessment. Clin Pharmacol Ther 2003;74:513-15.

3 Miller FG, Grady C. The ethical challenge of infection-inducing challenge experiments. Clin Infect Dis 2001;33:1028-33.

4 Mosely JB, O'Malley K, Petersen NJ, et al. A controlled trial of arthroscopic surgery for osteoarthritis of the knee. NEJM 2002;347:81-8.

5 Podolsky S. Quintessential Beecher: surgery as placebo: a quantitative study of bias. JAMA 1961;176:1102-7.

6 Jonas H. Philosophical Reflections on Experimenting with Human Subjects. Daedalus 1969;98:219-47.

7 Department of Health and Human Services. Criteria for IRB approval of research. Code of Federal Regulations: http://www.hhs.gov/ohrp/regulations-and-policy/ regulations/45-cfr-46/\#46.111. 